\title{
UNIQUENESS AND THE CONVERGENCE OF SUCCESSIVE APPROXIMATIONS. II
}

\author{
A. D. ZIEBUR
}

It has long been known that the uniqueness of the solution of an ordinary differential problem of the type of (1) below and the convergence of sequences of successive approximations (Picard sequences) to solutions are logically independent. Thus Brauer and Sternberg [2] list examples (due to Müller and Dieudonné) in which there is uniqueness but not convergence or convergence but not uniqueness. Nevertheless, uniqueness and convergence are closely related, and we have recently shown [10], for a special type of equation, and then only in the case $n=1$, that we can associate with a given differential problem another problem in such a way that the uniqueness of the solution of the associated problem guarantees the convergence of sequences of successive approximations to the (necessarily unique) solution of the original problem. In this note we remove the restriction to a "special type" of equation and extend the results to systems of $n$ equations, where $n$ is an arbitrary positive integer.

If the functions $f_{i}$ in problem (1) satisfy a Lipschitz condition, then the problem has a unique solution, and this solution is the limit of sequences of successive approximations. Over the years, many weaker "Lipschitz-like" conditions which guarantee uniqueness and convergence have been found. Presently known conditions of this kind guarantee the uniqueness of the solution of our associated problem, and in this sense our results are generalizations of previous work. We discuss this point in more detail after we state our main theorem.

We consider a differential problem of order $n$, that is, a system of $n$ differential equations with a given initial condition:

$$
\begin{aligned}
x_{i}^{\prime} & =f_{i}\left(t, x_{1}, \cdots, x_{n}\right), \quad i \in N, \quad \text { and } \quad\left(x_{1}, \cdots, x_{n}\right) \\
& =\left(a_{1}, \cdots, a_{n}\right) \quad \text { when } t=0 .
\end{aligned}
$$

(Here $N$ denotes the set of positive integers $\{1, \cdots, n\}$; the nature of our work is such that vector notation is of no advantage.) We assume that the functions $f_{i}, i \in N$, are continuous in an $(n+1)$-dimensional region

$$
R=\left\{\left(t, x_{1}, \cdots, x_{n}\right): t \in[0, h],\left|x_{i}-b_{i}\right| \leqq k, \quad i \in N\right\},
$$

where $h$ and $k$ are two given positive numbers. Let us set

Received by the editors November 27, 1963. 
$M=\max \left|f_{i}\left(t, x_{1}, \cdots, x_{n}\right)\right|,\left(t, x_{1}, \cdots, x_{n}\right) \in R, i \in N$, and then let $m=\min \{h, k / M\}$. In the definition of $R$, replace $h$ with $m$ and call the resulting region $R^{*}$. Now suppose that the functions $\xi_{10}, \cdots, \xi_{1 n}$ are defined in $[0, m]$ and are such that for each $t \in[0, m]$ the point $\left(t, \xi_{10}(t), \cdots, \xi_{n 0}(t)\right) \in R^{*}$. These functions initiate the Picard sequence $\left\{\xi_{1}, \cdots, \xi_{n v}\right\}$, the rest of whose terms are defined by the equations

$$
\begin{gathered}
\xi_{i(\nu+1)}(t)=a_{i}+\int_{0}^{t} f_{i}\left(s, \xi_{1 \nu}(s), \cdots, \xi_{n \nu}(s)\right) d s \\
i \in N, \nu=0,1, \cdots
\end{gathered}
$$

If our Picard sequence converges, its limit will constitute a solution of (1), so we now seek conditions that will guarantee the convergence of this sequence. Thus if we set

$$
\lambda_{i}(t)=\lim \inf \xi_{i \nu}(t) \text { and } \Lambda_{i}(t)=\lim \sup \xi_{i \nu}(t), \quad i \in N,
$$

we are looking for conditions under which we can assert that $\lambda_{i}(t)$ $=\Lambda_{i}(t)$ for each $t \in[0, m]$ and for each $i \in N$. From (2) we see that

$$
\Lambda_{i}(t)=a_{i}+\lim \sup \int_{0}^{t} f_{i}\left(s, \xi_{1 v}(s), \cdots, \xi_{n v}(s)\right) d s, \quad i \in N .
$$

According to Fatou's Lemma, we may bring the symbols lim sup inside the integral sign if we replace the sign of equality with $\leqq$ :

$$
\Lambda_{i}(t) \leqq a_{i}+\int_{0}^{t} \lim \sup f_{i}\left(s, \xi_{1 \nu}(s), \cdots, \xi_{n \nu}(s)\right) d s, \quad i \in N .
$$

By similar reasoning, we obtain the inequalities

(4) $\quad \lambda_{j}(t) \geqq a_{j}+\int_{0}^{t} \lim \inf f_{j}\left(s, \xi_{1 \nu}(s), \cdots, \xi_{n \nu}(s)\right) d s, \quad j \in N$.

If the functions $f_{i}, i \in N$, were monotonic, we could bring the symbols lim sup and lim inf inside the parentheses in (3) and (4). But we have made no assumption of monotonicity regarding these functions, and so we shall not proceed further with inequalities (3) and (4) until we have "embedded" each $f_{i}$ in a monotone function $F_{i}$ as described below.

These monotone functions will be functions of $2 n+1$ variables, defined in the region

$$
\begin{aligned}
S=\left\{\left(t, y_{1}, \cdots, y_{n}, z_{1}, \cdots, z_{n}\right):\right. & \\
& \left.t \in[0, m],\left|y_{i}-a_{i}\right| \leqq k,\left|z_{i}-a_{i}\right| \leqq k, \quad i \in N\right\} .
\end{aligned}
$$


To find the value of $F_{i}$ at a given point $\left(t, y_{1}, \cdots, y_{n}, z_{1}, \cdots, z_{n}\right)$ of $S$, we proceed in $n$ steps. In what follows we assume that $\left|x_{j}-a_{j}\right| \leqq k$ for each $j \in N$. We first set

$$
\begin{aligned}
f_{i 1}\left(t, y_{1}, x_{2}, \cdots, x_{n}, z_{1}\right) & =\max _{z_{1} \leqq x_{1} \leqq y_{1}} f_{i}\left(t, x_{1}, x_{2}, \cdots, x_{n}\right) & \text { if } z_{1} \leqq y_{1}, \\
& =\min _{y_{1} \leqq x_{1} \leqq z_{1}} f_{i}\left(t, x_{1}, x_{2}, \cdots, x_{n}\right) & \text { if } y_{1} \leqq z_{1} .
\end{aligned}
$$

Then we take

$f_{i 2}\left(t, y_{1}, y_{2}, x_{3}, \cdots, x_{n}, z_{1}, z_{2}\right)$

$$
\begin{array}{ll}
=\max _{z_{2} \leqq x_{2} \leqq y_{2}} f_{i 1}\left(t, y_{1}, x_{2}, \cdots, x_{n}, z_{1}\right) & \text { if } z_{2} \leqq y_{2}, \\
=\min _{y_{2} \leqq x_{2} \leqq z_{2}} f_{i 1}\left(t, y_{1}, x_{2}, \cdots, x_{n}, z_{1}\right) & \text { if } y_{2} \leqq z_{2},
\end{array}
$$

and we continue this process in the obvious way to find

$$
F_{i}\left(t, y_{1}, \cdots, y_{n}, z_{1}, \cdots, z_{n}\right) .
$$

It is a matter of straightforward calculation to verify that:

(i) $F_{i}$ is continuous in $S$.

(ii) If $\left(t, x_{1}, \cdots, x_{n}\right) \in R^{*}$, then $\left(t, x_{1}, \cdots, x_{n}, x_{1}, \cdots, x_{n}\right) \in S$, and

$$
F_{i}\left(t, x_{1}, \cdots, x_{n}, x_{1}, \cdots, x_{n}\right)=f_{i}\left(t, x_{1}, \cdots, x_{n}\right) .
$$

(iii) $F_{i}\left(t, y_{1}, \cdots, y_{n}, z_{1}, \cdots, z_{n}\right)$ is nondecreasing in each $y_{j}$ and nonincreasing in each $z_{j}, j \in N$.

Because of property (5) (ii), we can write inequalities (3) as

$$
\begin{aligned}
\Lambda_{i}(t) \leqq a_{i}+\int_{0}^{t} \lim \sup F_{i}\left(s, \xi_{1 \nu}(s), \cdots, \xi_{n v}(s),\right. & \\
& \left.\xi_{1 v}(s), \cdots, \xi_{n \nu}(s)\right) d s, \quad i \in N .
\end{aligned}
$$

Now we use the monotonicity property (5) (iii) of $F_{i}$ to get the first $n$ of inequalities (6) below; the remaining $n$ are obtained from inequalities (4) by a similar argument:

$$
\begin{array}{r}
\Lambda_{i}(t) \leqq a_{i}+\int_{0}^{t} F_{i}\left(s, \Lambda_{1}(s), \cdots, \Lambda_{n}(s), \lambda_{1}(s), \cdots, \lambda_{n}(s)\right) d s, \\
i \in N,
\end{array}
$$

$$
\lambda_{j}(t) \geqq a_{j}+\int_{0}^{t} F_{j}\left(s, \lambda_{1}(s), \cdots, \lambda_{n}(s), \Lambda_{1}(s), \cdots, \Lambda_{n}(s)\right) d s,
$$


At this point we turn to Opial's generalization of Bellman's lemma [8]. Kamke [4] has shown that the differential problem consisting of the $2 n$ equations

$$
\begin{aligned}
y_{i}^{\prime}=F_{i}\left(t, y_{1}, \cdots, y_{n}, z_{1}, \cdots, z_{n}\right), & i \in N, \\
z_{j}^{\prime}=F_{j}\left(t, z_{1}, \cdots, z_{n}, y_{1}, \cdots, y_{n}\right), & j \in N,
\end{aligned}
$$

and the initial conditions

$$
\left(y_{1}, \cdots, y_{n}, z_{1}, \cdots, z_{n}\right)=\left(a_{1}, \cdots, a_{n}, a_{1}, \cdots, a_{n}\right) \quad \text { when } t=a
$$

has an extreme solution $\left(\Omega_{1}, \cdots, \Omega_{n}, \omega_{1}, \cdots, \omega_{n}\right)$, defined in the interval $[0, m]$ and with the property that if $\left(\eta_{1}, \cdots, \eta_{n}, \zeta_{1}, \cdots, \zeta_{n}\right)$ is any solution of the problem, then for each $t \in[0, m]$ we have, for each $i \in N, \omega_{i}(t) \leqq \eta_{i}(t) \leqq \Omega_{i}(t)$ and $\omega_{i}(t) \leqq \zeta_{i}(t) \leqq \Omega_{i}(t)$. (Actually, in order to transform our system to agree exactly with Kamke's wording, we must make the substitution $w_{j}=-z_{j}, j \in N$, but this change is trivial.) Opial's theorem says that since the functional values $\lambda_{i}(t), \Lambda_{i}(t), i \in N$, satisfy inequalities (6), they are bounded by the values of the extreme solution of the differential problem (7)-(8). More precisely, for each $t \in[0, m]$ we have

$$
\omega_{i}(t) \leqq \lambda_{i}(t) \leqq \Lambda_{i}(t) \leqq \Omega_{i}(t), \quad i \in N .
$$

Inequalities (9) give us bounds on the upper and lower limits of the Picard sequence $\left\{\xi_{1 v}(t), \cdots, \xi_{n \nu}(t)\right\}$. We may also use them to derive a criterion for determining when these limits are equal. Let us note that if $\left(\xi_{1}, \cdots, \xi_{n}\right)$ is any solution of our original differential problem (1), then by (5) (ii), $\left(\xi_{1}, \cdots, \xi_{n}, \xi_{1}, \cdots, \xi_{n}\right)$ is a solution of the problem (7)-(8). From this fact we infer that if the problem (7)-(8) has a unique solution, then the problem (1) has a unique solution. This solution of (1) then determines the solution of $(7)-(8)$; it must have the form $\left(\xi_{1}, \cdots, \xi_{n}, \xi_{1}, \cdots, \xi_{n}\right)$. Since it is the only solution, this solution must be the extreme solution $\left(\Omega_{1}, \cdots, \Omega_{n}, \omega_{1}, \cdots, \omega_{n}\right)$; in other words, $\omega_{i}(t)=\Omega_{i}(t), i \in N$. Therefore we see from (9) that $\lambda_{i}(t)=\Lambda_{i}(t)$, and we have proved the following theorem.

THEOREM. If the differential problem (7)-(8) has a unique solution, then the differential problem (1) has a unique solution in the interval $[0, m]$, and the Picard sequence $\left\{\xi_{1 v}, \cdots, \xi_{n v}\right\}$ converges to that solution.

LaSalle [6] studied the convergence of sequences of successive approximations to solutions of differential problems of the type of (1), in which he also assumed that each $f_{i}\left(t, x_{1}, \cdots, x_{n}\right)$ is nondecreasing 
in $x_{1}, \cdots, x_{n}$. We may note that when $f_{i}\left(t, x_{1}, \cdots, x_{n}\right)$ is nondecreasing in $x_{1}, \cdots, x_{n}$, then our corresponding function $F_{i}$ is simply given by the equation $F_{i}\left(t, y_{1}, \cdots, y_{n}, z_{1}, \cdots, z_{n}\right)$ $=f_{i}\left(t, y_{1}, \cdots, y_{n}\right)$. If this equation holds for each $i \in N$, then the differential problem consisting of the $2 n$ equations (7) and initial conditions (8) is merely two copies of (1). In particular, then, it has a unique solution if, and only if, (1) has a unique solution, and we have the following corollary to our main theorem.

Corollary 1. If, for each $i \in N, f_{i}\left(t, x_{1}, \cdots, x_{n}\right)$ is nondecreasing in $x_{1}, \cdots, x_{n}$, and if the differential problem (1) has a unique solution, then the Picard sequence $\left\{\xi_{1 \nu}, \cdots, \xi_{n \nu}\right\}$ converges to that solution.

Well-known conditions that guarantee uniqueness of, and convergence of Picard sequences to, solutions of (1) consist of one or more sets of inequalities of the form

$$
\begin{aligned}
\left|f_{i}\left(t, x_{1}, \cdots, x_{n}\right)-f_{i}\left(t, \tilde{x}_{1}, \cdots, \tilde{x}_{n}\right)\right| & \\
& \leqq L\left(t, \max \left|x_{j}-\tilde{x}_{j}\right|, j \in N\right), \quad i \in N .
\end{aligned}
$$

For example, in the ordinary Lipschitz condition, $L(t, r)=p r$, where $p$ is a positive number. There is a great deal of work devoted to finding weaker functions $L$ that guarantee uniqueness and convergence; Santoro [9] lists a number of recent results. In presently known cases, each $L(t, r)$ is nondecreasing in $r$. The next corollary to our theorem shows that when we add this monotonicity condition to any assumption about the functions $L$ that guarantee uniqueness, then we obtain conditions that also guarantee the convergence of Picard sequences.

COROLlaRY 2. If one or more sets of inequalities of the type of (10) guarantee the uniqueness of the solution of a differential problem, and if each $L(t, r)$ is nondecreasing in $r$, then the convergence of sequences of successive approximations is also guaranteed.

This assertion is easy to prove. It is a straightforward, but tedious, matter to verify (using the monotonicity that we have assumed for a given $L$ ) that for each of our monotone functions $F_{i}$, we have

$$
\begin{aligned}
\mid F_{i}\left(t, y_{1}, \cdots\right. & \left., y_{n}, z_{1}, \cdots, z_{n}\right)-F_{i}\left(t, \tilde{y}_{1}, \cdots, \tilde{y}_{n}, \tilde{z}_{1}, \cdots, \tilde{z}_{n}\right) \mid \\
& \leqq L\left(t, \max \left\{\left|y_{j}-\tilde{y}_{j}\right|,\left|z_{j}-\tilde{z}_{j}\right|, j \in N\right\}\right), \quad i \in N .
\end{aligned}
$$

We are assuming that sets of inequalities of this sort guarantee the uniqueness of solutions of a differential problem, so problem (7)-(8) has a unique solution. Hence our theorem tells us that Picard sequences converge to the solution of (1). 
References [1], [5], and [7] are devoted to showing that certain uniqueness conditions due to Krasnosel'skiĭ and Krein are also sufficient to guarantee the convergence of sequences of successive approximations. These uniqueness conditions can be considered to be two sets of inequalities of the type of $(10)$ in which each $L(t, r)$ is nondecreasing in $r$. Therefore, according to our Corollary 2, these uniqueness conditions automatically guarantee convergence.

There are uniqueness conditions of the form of (10) (see [3, p. 51], for example) in which $L(t, r)$ is not necessarily nondecreasing. It is one of the interesting open questions of this subject whether such a condition can also guarantee convergence of Picard sequences.

\section{REFERENCES}

1. F. Brauer, $A$ note on uniqueness and convergence of successive approximations, Canad. Math. Bull. 2 (1959), 5-8.

2. F. Brauer and S. Sternberg, Local uniqueness, existence in the large, and the convergence of successive approximations, Amer. J. Math. 80 (1958), 421-430.

3. E. A. Coddington and N. Levinson, Theory of ordinary differential equations, McGraw-Hill, New York, 1955.

4. E. Kamke, Zur Theorie der Systeme gewöhnlicher Differentialgleichungen. II, Acta Math. 58 (1932), 57-85.

5. O. Kooi, The method of successive approximations and a uniqueness theorem of Krasnosel'skit and Kretn in the theory of differential equations, Nederl. Akad. Wetensch. Proc. Ser. A. 61 = Indag. Math. 20 (1958), 322-327.

6. J. LaSalle, Uniqueness theorems and successive approximations, Ann. of Math. (2) 50 (1949), 722-730.

7. W. A. J. Luxemburg, On the convergence of successive approximations in the theory of ordinary differential equations, Canad. Math. Bull. 1 (1958), 9-20.

8. Z. Opial, Sur un système d'inégalités intégrales, Ann. Polon. Math. 3 (1957), 200-209.

9. P. Santoro, Convergenza delle approssimazioni successive per le equazioni differenziali di ordine n, Riv. Mat. Univ. Parma (2) 2 (1961), 301-312.

10. A. D. Ziebur, Uniqueness and the convergence of successive approximations, Proc. Amer. Math. Soc. 13 (1962), 899-903.

Harpur College 